Preprint typeset in JHEP style - HYPER VERSION

\title{
Localization of Matters on Anti-de Sitter Thick Branes
}

\author{
Yu-Xiao Liu, Heng Guo, Chun-E Fu, Ji-Rong Ren \\ Institute of Theoretical Physics, Lanzhou University, Lanzhou 730000, People's Republic \\ of China \\ E-mail: liuyx@lzu.edu.cn, guoh2009@lzu.cn, fuche08@lzu.cn, renjr@lzu.edu.cn
}

\begin{abstract}
By presenting the mass-independent potentials of the Kaluza-Klein (KK) modes in the corresponding Schrödinger equations, we investigate the localization and mass spectra of various bulk matter fields on an AdS thick brane. For a spin 0 scalar $\Phi$ coupled with itself and the domain-wall-forming field $\phi$ via a coupling potential $V=$ $\left(\lambda \phi^{2}-u^{2}\right) \Phi^{2}+\tau \Phi^{4}$, the localization and spectrum are decided by a critical coupling constant $\lambda_{0}$. When $\lambda>\lambda_{0}$, the potential of the scalar KK modes in the corresponding Schrödinger equation tends to infinite when far away from the brane, which results in that there exist infinite discrete scalar bound KK states, and the massless modes could be trapped on the AdS brane by fine-tuning of parameters. When $\lambda<\lambda_{0}$, the potential of the scalar KK modes tends to negative infinite when far away from the brane, hence there does not exist any scalar bound KK state. For a spin 1 vector, the situation is same like the scalar with a coupling constant $\lambda>\lambda_{0}$, but the zero mode can not be localized on the brane. For a spin 1/2 fermion, we introduce the usual Yukawa coupling $\eta \bar{\Psi} \phi \Psi$, and find that the localization of the fermion is decided by a critical coupling constant $\eta_{0}$. For $\eta>\eta_{0}$, the four-dimensional massless left chiral fermion and massive Dirac fermions consisted of the pairs of coupled left-hand and right-hand KK modes could be localized on the AdS brane, and the massive Dirac fermions have a set of discrete mass spectrum. While for the case $0<\eta<\eta_{0}$, no four-dimensional Dirac fermion can be localized on the AdS brane.
\end{abstract}

Keywords: Large Extra Dimensions, Field Theories in Higher Dimensions, Brane world.

\footnotetext{
${ }^{*}$ Corresponding author.
} 


\section{Contents}

1. Introduction 1

2. Review of anti-de Sitter thick brane 3

3. Localization of various matters on an AdS thick brane 1

3.1 Spin-0 scalar fields

3.2 Spin-1 vector fields 10

3.3 Spin- $1 / 2$ fermion fields

4. Conclusion 17

\section{Introduction}

The proposal that our observed four-dimensional universe as a sub-manifold is embedding in a higher dimensional space, has received a considerable attention. Branes naturally appear in string/M-theory context and provide a novel mode for discussing phenomenological and cosmological issues related to extra dimensions. The idea that extra dimensions may not be compact [1, 2, 3, 4, 5] or large [6, 7] can supply new insights for solving gauge hierarch problem [7] and cosmological constant problem [1, 3, 8]. The framework of brane scenarios is that gravity is free to propagate in all dimensions, however all matter fields are confined to a 3-brane with no contradiction with present gravitational experiments [1, 2, [7]. In Randall-Sundrum (RS) brane model [4], the internal manifold does not need to be compactified to the Planck scale any more, it can be large, or even infinite non-compact. But in this model, the branes are very idealized because of their thickness is neglected. It is also widely considered that the most fundamental theory would have a minimal length scale.

Since thick brane scenarios based on gravity coupled to scalars have been constructed, more and more authors have investigated the thick brane scenario in higher dimensional space-time [9, 10, 11, 12, 13, 14, 15, 16, 17, 18, 19]. In these models, there is an interesting feature that we can obtain branes naturally without introducing them by hand in the action of the theory [9]. In this scenario the scalar fields do not play the role of bulk fields but provide the "material" from which the thick branes are made of. For a comprehensive review on thick branes please see Ref. [20].

In brane world scenarios, there is an important problem: whether various bulk fields could be localized on the brane by a natural mechanism. Generally, massless scalar fields [21] and graviton [4] can be localized on brane of different types. Vector fields can be localized on the RS brane in some higher-dimensional cases [22] or on the thick $d S$ brane 
and Weyl thick brane 23]. It is important that whether the fermions could be localized on the thick branes. Without introducing the scalar-fermion coupling, fermions do not have normalizable zero modes in five and six dimensions [21, 22, 23, 24, 25, 26, 27, 28, 29, 30, 31, 32, 33, 34, 35, 36, 37, 38, 39]. In some cases, with scalar-fermion coupling, one can obtain a single bound state and a continuous gapless spectrum of massive fermion Kaluza-Klein (KK) states [23, 40]. In some other brane models, one can obtain finite discrete KK states (mass gap) and a continuous gapless spectrum starting at a positive $m^{2}$ [41, 42, 43].

In Ref. [42], the authors have investigated the localization and mass spectra of various matter fields on the symmetric and asymmetric dS thick branes. For scalar and vector fields, the potentials of the KK modes in the corresponding Schrödinger equations are the modified Pöschl-Teller potentials. In this case, there exist finite mass gap and a series of continuous spectrum. For fermions, when one introduces the usual Yukawa coupling, there exists no mass gap but a continuous gapless spectrum of KK states. While in Ref. [43], the same problem of various matter fields on a family of thick brane configurations in a pure geometric 5-dimensional spacetime was studied. It was shown that, for a class of brane configurations, there exists a continuum gapless spectrum of KK modes with any $m^{2}>0$ for scalars, vectors and ones of left chiral and right chiral fermions. However, for a special of brane configuration, there exist mass gap and a series of continuous spectrum starting at positive $m^{2}$ for scalars, vectors and fermions.

Recently, "Locally localized gravity" solutions with $\mathrm{AdS}_{4}$ and $\mathrm{dS}_{4}$ brane cosmology has been presented in Ref. [25]. In the case of a brane with $\mathrm{AdS}_{4}$ cosmology, the warp factor grows exponentially when far from the brane. However, when closes to the brane, the metric behaves like the decreasing warped metric associated with a Minkowski brane, and it is this local behavior that is responsible for confining gravity to the brane [25, 44, 45]. Confinement of the fermion zero mode on the $\mathrm{AdS}_{4}$ brane has been discussed in Ref. [25], and the localization conditions for left-handed and right-handed fermion zero modes were obtained. In this paper, in order to reveal the rich structures of the $\mathrm{AdS}_{4}$ brane from other point of view, we would like to investigate further the localization problem of various spin massive mater fields (scalars, vectors and fermions) on the brane. We find the results especially for scalars and fermions are very nontrivial and have not been reported in the literature: For a spin-0 scalar $\Phi$ coupled with itself and the background scalar $\phi$ via the coupling potential $\left(\lambda \phi^{2}-u^{2}\right) \Phi^{2}+\tau \Phi^{4}$, we find that the localization characteristic is related closely to a critical coupling constant $\lambda_{0}$. When $\lambda>\lambda_{0}$, there exist a series of discrete bound KK modes and the zero mode could be trapped on the AdS thick brane by finetuning of parameters. However, when $\lambda<\lambda_{0}$, there does not exist any bound KK modes. For a spin- 1 vector, there exist infinite massive bound KK modes, but the massless mode could not be trapped on the AdS brane. For a fermion coupled with the background scalar via the usual Yukawa coupling $\eta \bar{\Psi} \phi \Psi$, in order to get a series of four-dimensional massive Dirac fermions and a massless left chiral fermion, the coupling constant $\eta$ should larger than a critical coupling constant $\eta_{0}$.

The organization of the paper is as follows: In Sec. 2, we first review the AdS thick brane in 5-dimensional space-time. Then, in Sec. 3, we study the localization and mass spectra of various bulk fields on the AdS thick brane by presenting the potentials of the 
corresponding Schrödinger problem. For scalar fields, we introduce the Higgs coupling and discuss the spectra of scalars in detail. For vector fields, we obtain infinite bound states and infinite mass gaps. For spin 1/2 fermions, we introduce the usual Yukawa coupling and discuss the spectra of fermions in detail. Finally, our conclusion is given in Sec. 6 .

\section{Review of anti-de Sitter thick brane}

Let us consider thick branes arising from a real scalar field $\phi$ with a scalar potential $V(\phi)$. The action for such a system can be expressed as

$$
S=\int d^{5} x \sqrt{-g}\left[\frac{1}{2 \kappa_{5}^{2}} R-\frac{1}{2} g^{M N} \partial_{M} \phi \partial_{N} \phi-V(\phi)\right],
$$

where $R$ is the scalar curvature and $\kappa_{5}^{2}=8 \pi G_{5}$ with $G_{5}$ the 5 -dimensional Newton constant. Here we set $\kappa_{5}=1$. The line-element for a 5 -dimensional spacetime is assumed as

$$
d s^{2}=g_{M N} d x^{M} d x^{N}=\mathrm{e}^{2 A(y)} \hat{g}_{\mu \nu}(x) d x^{\mu} d x^{\nu}+d y^{2},
$$

where $\mathrm{e}^{2 A(y)}$ is the warp factor and $y$ stands for the extra coordinate. We suppose that $\hat{g}_{\mu \nu}$ is some general 4-dimensional metric. The scalar field is considered to be a function of $y$ only, i.e., $\phi=\phi(y)$. In the model, the potential could provide a thick brane realization, and the soliton configuration of the scalar field dynamically generates the domain wall with warped geometry. The field equations generated from the action (2.1) with the ansatz (2.2) reduce to the following coupled nonlinear differential equations

$$
\begin{aligned}
\phi^{2} & =-3 A^{\prime \prime}-\gamma e^{-2 A}, \\
V(\phi) & =\frac{1}{2} \phi^{\prime 2}-6 A^{\prime 2}+2 \gamma e^{-2 A}, \\
\frac{d V(\phi)}{d \phi} & =\phi^{\prime \prime}+4 A^{\prime} \phi^{\prime},
\end{aligned}
$$

where the prime denotes derivative with respect to $y$ and $\gamma$ is some constant such that $\hat{G}_{\mu \nu}=\gamma \hat{g}_{\mu \nu}$. In the case of an AdS brane cosmology, $\gamma$ is negative, while for a dS brane cosmology we have $\gamma>0$. Minkowski space corresponds to the case $\gamma=0$.

We consider a trial warp factor of the form [25],

$$
e^{A(y)}=a \cosh (c y)+b \operatorname{sech}(c y),
$$

where $a$ and $b$ are dimensionless constants and $c=r \sqrt{\frac{|\gamma|}{3}}$ with $r$ a positive constant. Then Eq. (2.3) becomes

$$
\phi^{\prime 2}=\frac{-|\gamma|}{[a \cosh (c y)+b \operatorname{sech}(c y)]^{2}}\left\{\frac{\gamma}{|\gamma|}+r^{2}\left[a(a+4 b)-4 a b \operatorname{sech}^{2}(c y)-b^{2} \operatorname{sech}^{4}(c y)\right]\right\} \cdot(2.7
$$

If the r.h.s. of the above equation is always positive and to facilitate an analytic solution, we should impose the relation

$$
-\frac{\gamma}{|\gamma|}=r^{2}\left(4 a^{2}+a(a+4 b)\right) .
$$


Then Eq. (2.7) reduces to

$$
\phi^{\prime 2}=|\gamma| r^{2} \operatorname{sech}^{2}(c y)\left(\frac{2 a+b \operatorname{sech}^{2}(c y)}{a+b \operatorname{sech}^{2}(c y)}\right)^{2},
$$

which can be solved as [25]

$$
\phi(y)= \pm \sqrt{3}\left[\arctan \sinh (c y)+\sqrt{\frac{a}{a+b}} \arctan \left(\sqrt{\frac{a}{a+b}} \sinh (c y)\right)\right] .
$$

The potential $V$ can easily be expressed as a function of $y$ :

$$
\begin{aligned}
V(y)=\frac{r^{2}|\gamma|}{\left[a+b \operatorname{sech}^{2}(c y)\right]^{2}} & {\left[-2 a(3 a+2 b) \operatorname{sech}^{2}(c y)-2 b(a+b) \operatorname{sech}^{4}(c y)\right.} \\
& \left.+\frac{5}{2} b^{2} \operatorname{sech}^{6}(c y)-2 a^{2}\right]
\end{aligned}
$$

Generally, $V$ can not be written analytically in term of standard function of $\phi$, however $V$ can always be expressed numerically as a well-defined function of $\phi$.

In this paper we would like to investigate the AdS brane cosmology, so $\gamma$ is negative, and by Eq. (2.8), this is equivalent to requiring

$$
5 a^{2}+4 a b>0
$$

\section{Localization of various matters on an AdS thick brane}

In this section, we will investigate whether various bulk mater fields such as spin-0 scalars, spin-1 vectors and spin- $1 / 2$ fermions can be localized on the AdS thick brane by means of the gravitational interaction. Certainly, we have implicitly assumed that various bulk mater fields considered below make little contribution to the bulk energy so that the solutions given in previous section remain valid even in the presence of bulk fields. We will also discuss the spectra of various mater fields on the AdS thick brane by presenting the potential of the corresponding Schrödinger equation for KK modes of various matter fields.

In order to get mass-independent potentials, we will follow Ref. [4] and change the metric given in (2.2) to following one

$$
d s^{2}=\mathrm{e}^{2 A(z)}\left(\hat{g}_{\mu \nu} d x^{\mu} d x^{\nu}+d z^{2}\right)
$$

by performing the coordinate transformation

$$
d z=\mathrm{e}^{-A(y)} d y
$$

Then, we can obtain the following expression

$$
z(y)=\int e^{-A(y)} d y=\sqrt{\frac{1}{a(a+b)}} \frac{1}{c} \arctan \left[\sqrt{\frac{a}{a+b}} \sinh (c y)\right] .
$$


From this expression, it can be seen that $z \rightarrow \pm \frac{\pi}{2 c} \sqrt{\frac{1}{a(a+b)}}$, when $y \rightarrow \pm \infty$, so the range of $z$ is $-z_{\max }<z<z_{\max }$ with $z_{\max }$ defined by

$$
z_{\max }=\frac{\pi}{2 c} \sqrt{\frac{1}{a(a+b)}} .
$$

Inverting Eq. (3.3), $y(z)$ can be solved easily as

$$
y(z)=\frac{1}{c} \operatorname{arcsinh}\left[\sqrt{\frac{a+b}{a}} \tan (\sqrt{a(a+b)} c z)\right] .
$$

Due to this transformation, $\mathrm{e}^{A}$ and $\phi$ can be rewritten as functions of $z$ :

$$
\begin{gathered}
\mathrm{e}^{A(z)}=\frac{\sqrt{a}(a+b) \sec ^{2}(\sqrt{a(a+b)} c z)}{\sqrt{a+(a+b) \tan ^{2}(\sqrt{a(a+b)} c z)}}, \\
\phi= \pm \sqrt{3}\left[\arctan \left(\sqrt{\frac{a+b}{a}} \tan (\sqrt{a(a+b)} c z)\right)+a c z\right] .
\end{gathered}
$$

In next subsections, it can be seen that the mass-independent potentials can be obtained conveniently with the conformally metric (3.1).

\subsection{Spin-0 scalar fields}

Firstly, we will investigate localization of real scalar fields on the AdS brane which obtained in previous section. We start by considering the action of a real scalar $\Phi$ coupled to itself, gravity and the domain-wall-forming field $\phi$ [19]:

$$
S_{0}=\int d^{5} x \sqrt{-g}\left[-\frac{1}{2} g^{M N} \partial_{M} \Phi \partial_{N} \Phi-V(\Phi, \phi)\right]
$$

where $V(\Phi, \phi)$ is a coupling potential of $\Phi$ to itself and to the domain-wall-forming field $\phi$, which also called as Higgs potential, and $V(\Phi, \phi)$ should include $\Phi, \Phi^{2}, \Phi^{3}, \Phi^{4}$ and $(\phi \Phi)^{2}$ terms, but by considering a discrete symmetry, $\Phi$ and $\Phi^{3}$ terms can be eliminated. So we can set 19

$$
V(\Phi, \phi)=\left(\lambda \phi^{2}-u^{2}\right) \Phi^{2}+\tau \Phi^{4} .
$$

Using the conformal metric (3.1), the equation of motion derived from (3.8) reads as

$$
\frac{1}{\sqrt{-\hat{g}}} \partial_{\mu}\left(\sqrt{-\hat{g}} \hat{g}^{\mu \nu} \partial_{\nu} \Phi\right)+e^{-3 A} \partial_{z}\left(e^{3 A} \partial_{z} \Phi\right)-e^{2 A} U(\phi) \Phi=0
$$

where $U(\phi)$ is defined by

$$
\frac{\partial V(\Phi, \phi)}{\partial \Phi}=U(\phi) \Phi+\mathcal{O}\left(\Phi^{3}\right)
$$


and is calculated from Eq. (3.9) as

$$
U(\phi)=2\left(\lambda \phi^{2}-u^{2}\right) .
$$

Then, by making use of the KK decomposition $\Phi(x, z)=\sum_{n} \Phi_{n}(x) \chi_{n}(z) e^{-3 A / 2}$ and demanding $\Phi_{n}(x)$ satisfies the 4-dimensional massive Klein-Gordon equation

$$
\left(\frac{1}{\sqrt{-\hat{g}}} \partial_{\mu}\left(\sqrt{-\hat{g}} \hat{g}^{\mu \nu} \partial_{\nu}\right)-m_{n}^{2}\right) \Phi_{n}(x)=0
$$

we can obtain the equation for the scalar KK mode $\chi_{n}(z)$ :

$$
\left[-\partial_{z}^{2}+V_{0}(z)\right] \chi_{n}(z)=m_{n}^{2} \chi_{n}(z)
$$

which is a Schrödinger equation with the effective potential given by

$$
\begin{aligned}
V_{0}(z) & =\frac{3}{2} \partial_{z}^{2} A+\frac{9}{4}\left(\partial_{z} A\right)^{2}+e^{2 A} U(\phi) \\
& =\frac{3}{2} \partial_{z}^{2} A+\frac{9}{4}\left(\partial_{z} A\right)^{2}+2 e^{2 A}\left(\lambda \phi^{2}-u^{2}\right) .
\end{aligned}
$$

Here $m_{n}$ is the mass of the KK excitation. It is clear that $V_{0}(z)$ defined in (3.15) is a 4-dimensional mass-independent potential.

The full 5-dimensional action (3.8) reduces to the standard 4-dimensional action for the massive scalars

$$
S_{0}=-\frac{1}{2} \sum_{n} \int d^{4} x \sqrt{-\hat{g}}\left(\hat{g}^{\mu \nu} \partial_{\mu} \phi_{n} \partial_{\nu} \phi_{n}+m_{n}^{2} \Phi_{n}^{2}\right),
$$

when integrated over the extra dimension, in which it is required that Eq. (3.14) is satisfied and the following orthonormality condition is obeyed:

$$
\int_{-z_{\max }}^{z_{\max }} d z \chi_{m}(z) \chi_{n}(z)=\delta_{m n}
$$

We will consider two cases in this section. Firstly, we choose $U(\phi)=0$, that means the scalar field $\Phi$ does not couple to itself and the background scalar field $\phi(z)$. Secondly, we set $U(\phi)=2\left(\lambda \phi^{2}+u^{2}\right)$, and consider the scalar field $\Phi$ coupled to itself and $\phi(z)$.

For the AdS brane world solution (3.6) and $U(\phi)=0$, the potential corresponding to (3.15) is

$$
\begin{aligned}
V_{0}(z)=- & \frac{3 a c^{2}(a+b) \sec ^{2}(\sqrt{a(a+b)} c z)}{8[2 a+b-b \cos (2 \sqrt{a(a+b)} c z)]^{2}} \\
\times & \left\{-28 a^{2}-24 a b-14 b^{2}+\left(12 a^{2}+48 a b+17 b^{2}\right) \cos (2 \sqrt{a(a+b)} c z)\right. \\
& \left.-2 b(4 a+3 b) \cos (4 \sqrt{a(a+b)} c z)+3 b^{2} \cos (6 \sqrt{a(a+b)} c z)\right\} .
\end{aligned}
$$


It is clear that the potential $V_{0}$ has the asymptotic behavior: $V_{0}\left(z \rightarrow \pm z_{\max }\right) \rightarrow \infty$. For this potential, we can obtain the scalar zero mode $\chi_{0}(z)$ by setting $m_{0}=0$ :

$$
\chi_{0}(z) \propto \mathrm{e}^{\frac{3}{2} A(z)} .
$$

It can be seen that, when $z \rightarrow \pm z_{\max }$, this zero mode has the asymptotic behavior: $\chi_{0} \rightarrow$ $\pm \infty$, which is physically unacceptable. So it is clear that the zero mode could not be trapped on the AdS thick brane (see Fig. 11).

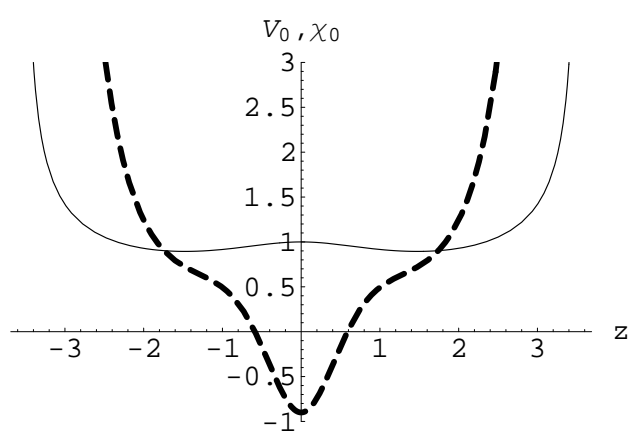

Figure 1: The shapes of the potential $V_{0}(z)$ (the dashed line) and the zero mode $\chi_{0}(z)$ (the thin line). The parameters are set as $\lambda=0, a=0.2, b=0.8, r=1, \gamma=-3$ and $u=0$.

However, from the potential $V_{0}(z)$ (3.18), we know that there are infinite bound KK modes. We can numerically solve Eq. (3.14), and the lower bound KK modes are plotted in Fig. 2 and the spectrum of the KK modes is listed as follows:

$$
m_{n}^{2}=\{0.31,1.87,3.25,5.32,7.69,10.52,13.74,17.38,21.44,25.93, \cdots\},
$$

where the parameters are set as $a=0.2, b=0.8, r=1, \gamma=-3$. So, the minimum mass of a 4-dimensional scalar with mass defined in Eq. (3.13) in background of an AdS brane cosmology is not zero, and all the 4-dimensional scalars must be massive and can be localized on the AdS thick brane. We plot the $m_{n}^{2}$ spectrum of the KK modes in Fig. 周.

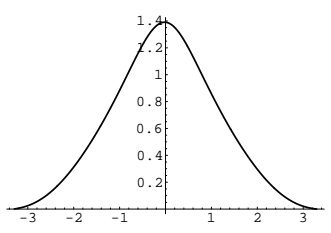

(a) $n=1$

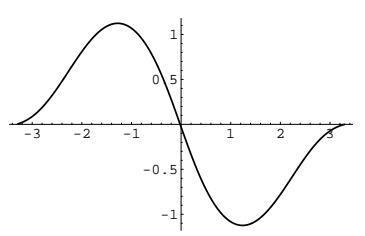

(b) $n=2$

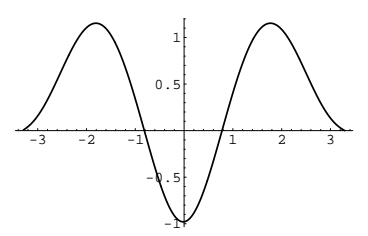

(c) $n=3$

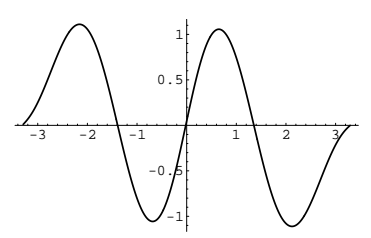

(d) $n=4$

Figure 2: The shapes of the scalar bound KK modes $\chi_{n}(z)$. The parameters are set as $\lambda=0$, $a=0.2, b=0.8, r=1, \gamma=-3$.

Next, we will consider the case $U(\phi)=2\left(\lambda \phi^{2}-u^{2}\right)$, namely, the scalar field $\Phi$ coupled 
to itself and the background scalar $\phi(z)$. The potential corresponding to (3.15) is

$$
\begin{aligned}
V_{0}(z)=- & \frac{3 a c^{2}(a+b) \sec ^{2}(\sqrt{a(a+b)} c z)}{8[2 a+b-b \cos (2 \sqrt{a(a+b)} c z)]^{2}} \\
& \times\left\{-28 a^{2}-24 a b-14 b^{2}+\left(12 a^{2}+48 a b+17 b^{2}\right) \cos (2 \sqrt{a(a+b)} c z)\right. \\
& \left.-2 b(4 a+3 b) \cos (4 \sqrt{a(a+b)} c z)+3 b^{2} \cos (6 \sqrt{a(a+b)} c z)\right\} \\
& \frac{2 a(a+b)^{2}\left\{3 \lambda\left[a c z+\arctan \left(\sqrt{\frac{a+b}{a}} \tan (\sqrt{a(a+b)} c z)\right)\right]^{2}-u^{2}\right\}}{\left[a+(a+b) \tan ^{2}(\sqrt{a(a+b)} c z)\right] \cos ^{4}(\sqrt{a(a+b)} c z)} .
\end{aligned}
$$

When $z \rightarrow \pm z_{\max }$, the even potential $V_{0}(z)$ can be reduced to

$$
V_{0}\left(z \rightarrow \pm z_{\max }\right) \rightarrow \frac{15 c^{2}+8\left[\lambda \phi\left(z_{\max }\right)^{2}-u^{2}\right]}{4 c^{2}\left(z \mp \frac{\pi}{2 \sqrt{a(a+b)} c}\right)^{2}}
$$

from which we find that the coupling constant $\lambda$ has a critical value $\lambda_{0}$, which can be expressed as

$$
\lambda_{0}=\frac{8 u^{2}-15 c^{2}}{8 \phi\left(z_{\max }\right)^{2}}
$$

For $\lambda>\lambda_{0}$ and $\lambda<\lambda_{0}$, the potential $V_{0}$ has different asymptotic behavior at $z \rightarrow \pm z_{\max }$. This can be seen from Fig. 3. For $\lambda>\lambda_{0}$, the potential $V_{0}\left(z \rightarrow \pm z_{\max }\right) \rightarrow+\infty$. While for $\lambda<\lambda_{0}, V_{0}\left(z \rightarrow \pm z_{\max }\right) \rightarrow-\infty$. Following, we will discuss the two cases of $\lambda>\lambda_{0}$ and $\lambda<\lambda_{0}$, respectively.

We first consider the case of $\lambda>\lambda_{0}$, for which the potential $V_{0}$ (3.21) has an infinite deep well (see Fig. 3(a)), and supports infinite discrete bound KK modes. When we set $\lambda>\lambda_{0}$ and fix the value of $\lambda$, if we choose a proper parameter $u$, the zero mode can be localized on the AdS brane, and if we increase the value of $u$, there will be exist the bound state solutions with $m_{n}^{2}<0$. In the following discussion, we will set the $\lambda>\lambda_{0}$ and fix the value of $\lambda$, and choose a proper $u$ for the zero mode localization. Then we can solve numerically Eq. (3.14), and the lower bound KK modes are plotted in Fig. 14 and the spectrum of the KK modes is listed as follows:

$$
m_{n}^{2}=\{0,5.867,10.058,13.593,18.122,22.651,27.568,32.883,38.602,44.726, \cdots\},
$$

where the parameters are set as $a=0.2, b=0.8, r=1, \gamma=-3, \lambda=1.460$ and $u=1.156$. So from the numerical solution, we know that the zero mode could be trapped on the AdS brane by fine-tuning of parameters, and all the 4-dimensional massive scalar fields can be trapped on the AdS thick brane. We plot the $m_{n}^{2}$ spectrum of the KK modes in Fig. 5. Comparing these two cases, we can see that the four-dimensional scalars would get larger mass when a coupling potential with a positive coupling constant is introduced. 


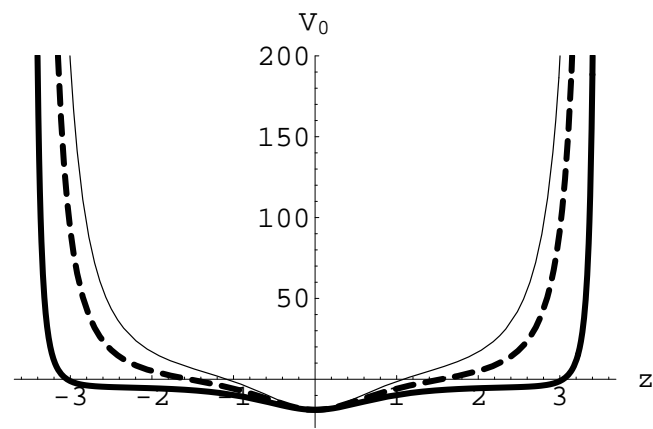

(a) $\lambda>\lambda_{0}$

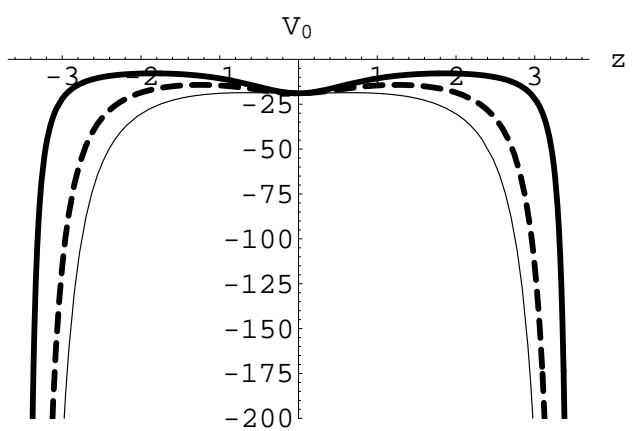

(b) $\lambda<\lambda_{0}$

Figure 3: The shapes of the potential $V_{0}(z)$. The parameters are set as $a=0.2, b=0.8, r=1$, $\gamma=-3$ and $u=3$. In Fig. (a), we set $\lambda>\lambda_{0}=0.460$, and $\lambda=0.560$ for the thick line, $\lambda=1.460$ for the dashed line, $\lambda=2.460$ for the thin line. In Fig. (b), we set $\lambda<\lambda_{0}$, and $\lambda=0.360$ for the thick line, $\lambda=-0.540$ for the dashed line, $\lambda=-1.540$ for the thin line.

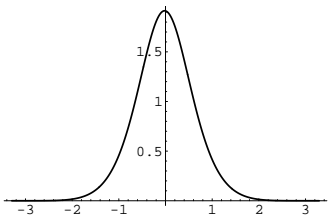

(a) $n=1$

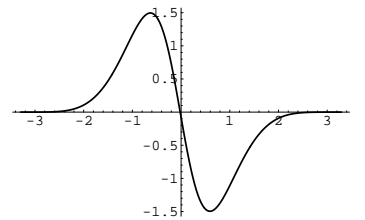

(b) $n=2$

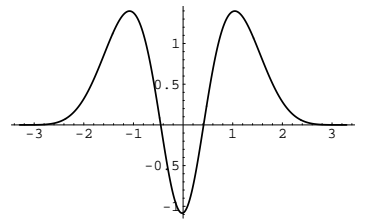

(c) $n=3$

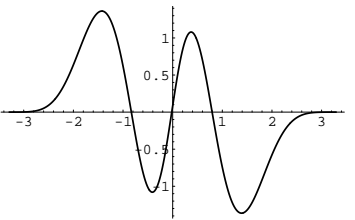

(d) $n=4$

Figure 4: The shapes of the scalar bound KK modes $\chi_{n}(z)$. The parameters are set as $a=0.2$, $b=0.8, r=1, \gamma=-3, \lambda=1.460$ and $u=1.156$.

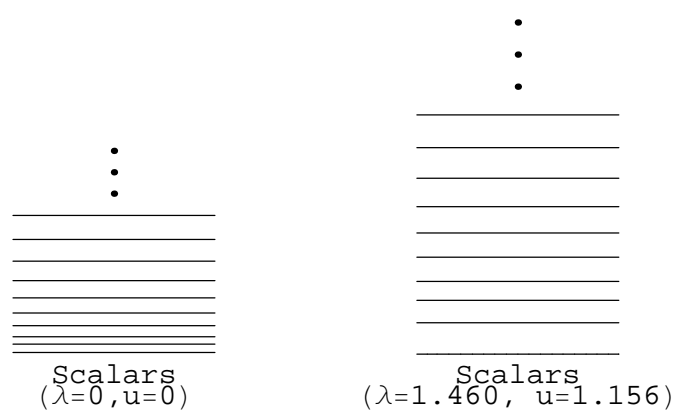

Figure 5: The $m_{n}^{2}$ spectrum of the scalar KK modes. The parameters are set as $a=0.2, b=0.8$, $r=1, \gamma=-3$.

For the case of $\lambda<\lambda_{0}$, the potential $V_{0}$ (3.21) has no well (see Fig. 3(b)], therefor all the scalar KK modes could not be localized on the AdS thick brane.

At the end of this section we will investigate the property of the coupling potential. 


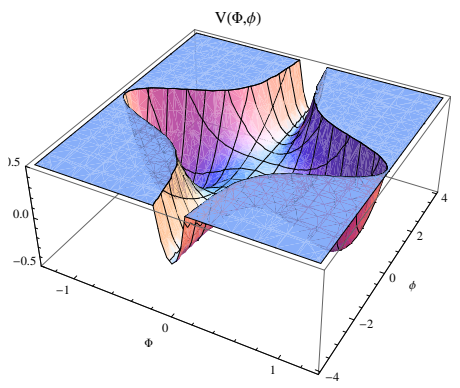

(a) $\lambda>\lambda_{m}$

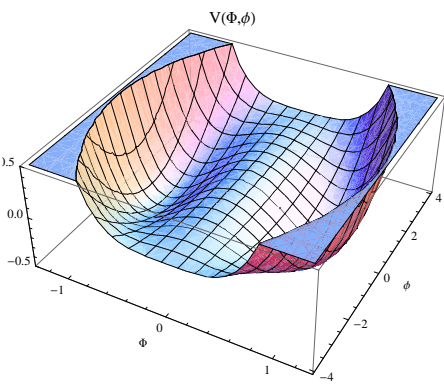

(b) $\lambda=\lambda_{m}$

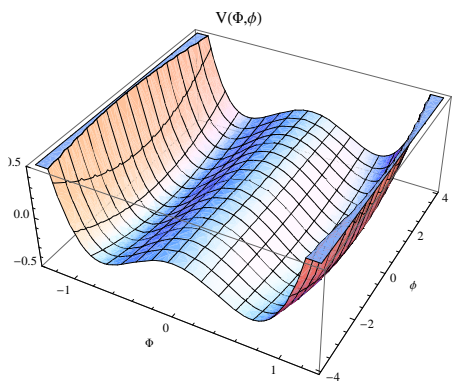

(c) $\lambda<\lambda_{m}$

Figure 6: The shapes of the potential $V(\Phi, \phi)$. The parameters are set as $a=0.2, b=0.8, r=1$, $\gamma=-3, \tau=1$ and $u=1.156$, from which we have $\lambda_{m}=0.086$. The range of $\phi$ is from $-\phi\left(z_{\max }\right)$ to $+\phi\left(z_{\max }\right)$. In Fig. (a), we set $\lambda=1.460>\lambda_{m}$. In Fig. (b), we set $\lambda=0.086=\lambda_{m}$. In Fig. (c), we set $\lambda=0.020<\lambda_{m}$.

From the expression (3.9), we can set $\lambda_{m} \phi\left(z_{\max }\right)^{2}-u^{2}=0$, and we can obtain

$$
\lambda_{m}=\frac{u^{2}}{\phi\left(z_{\max }\right)^{2}} .
$$

When $\lambda>\lambda_{m}$, we have plotted the coupling potential $V(\Phi, \phi)$ in Fig. 6(a). We can see that, if $\Phi \rightarrow 0$ as $z \rightarrow \pm z_{\max }$, then $(\phi, \Phi)=\left( \pm \phi\left(z_{\max }\right), 0\right)$ are the global minima of the potential. Because $\phi$ forms the domain wall and $\phi \sim 0$ inside the wall, so the leading term of $V(\Phi, \phi)$ is $-u^{2} \Phi^{2}$, which indicates that the $\Phi=0$ solution is unstable there [19]. When far from the domain wall, $\phi \rightarrow \pm \phi\left(z_{\max }\right)$ and $(\phi, \Phi)=\left( \pm \phi\left(z_{\max }\right), 0\right)$ are the global minima of the potential, so the $\Phi=0$ solution is stable there. When $\lambda=\lambda_{m}$ (see Fig. 6(b)]), the conclusion is the same as the case of $\lambda>\lambda_{m}$. When $\lambda<\lambda_{m}$ (see Fig. 6(c)), there is no stable solution. From Eqs. (3.23) and (3.25), we have $\lambda_{m}>\lambda_{0}$, so when $\lambda>\lambda_{m}\left(>\lambda_{0}\right)$, the $\Phi=0$ solution is stable at $z \rightarrow \pm z_{\max }$ and all the 4-dimensional scalars are localized on the thick brane.

\subsection{Spin-1 vector fields}

Secondly, we will investigate localization of spin-1 vector fields on the AdS brane. We begin with the 5-dimensional action of a vector field

$$
S_{1}=-\frac{1}{4} \int d^{5} x \sqrt{-g} g^{M N} g^{R S} F_{M R} F_{N S},
$$

where $F_{M N}=\partial_{M} A_{N}-\partial_{N} A_{M}$ is the field tensor as usual. From this action, one can obtain the equations of motion

$$
\frac{1}{\sqrt{-g}} \partial_{M}\left(\sqrt{-g} g^{M N} g^{R S} F_{N S}\right)=0 .
$$

By using of the background geometry (3.1), the equations of motion read as

$$
\begin{aligned}
\frac{1}{\sqrt{-\hat{g}}} \partial_{\nu}\left(\sqrt{-\hat{g}} \hat{g}^{\nu \rho} \hat{g}^{\mu \lambda} F_{\rho \lambda}\right)+\hat{g}^{\mu \lambda} e^{-A} \partial_{z}\left(e^{A} F_{4 \lambda}\right) & =0, \\
\partial_{\mu}\left(\sqrt{-\hat{g}} \hat{g}^{\mu \nu} F_{\nu 4}\right) & =0 .
\end{aligned}
$$


Because the fourth component $A_{4}$ has no zero mode in the effective $4 \mathrm{D}$ theory, we assume that it is $Z_{2}$-odd with respect to the extra dimension $z$. Furthermore, in order to consistent with the gauge invariant equation $\oint d z A_{4}=0$, we choose $A_{4}=0$ by using gauge freedom. Under the assumption, the action (3.26) becomes

$$
S_{1}=-\frac{1}{4} \int d^{5} x \sqrt{-g}\left\{g^{\mu \alpha} g^{\nu \beta} F_{\mu \nu} F_{\alpha \beta}+2 e^{-A} g^{\mu \nu} \partial_{z} A_{\mu} \partial_{z} A_{\nu}\right\} .
$$

Then, using the decomposition of the vector field $A_{\mu}(x, z)=\sum_{n} a_{\mu}^{(n)}(x) \rho_{n}(z) e^{-A / 2}$ and the orthonormality condition

$$
\int_{-z_{\max }}^{z_{\max }} d z \rho_{m}(z) \rho_{n}(z)=\delta_{m n}
$$

the action $(3.30)$ reduces to

$$
S_{1}=\sum_{n} \int d^{4} x \sqrt{-\hat{g}}\left(-\frac{1}{4} \hat{g}^{\mu \alpha} \hat{g}^{\nu \beta} f_{\mu \nu}^{(n)} f_{\alpha \beta}^{(n)}-\frac{1}{2} m_{n}^{2} \hat{g}^{\mu \nu} a_{\mu}^{(n)} a_{\nu}^{(n)}\right),
$$

where $f_{\mu \nu}^{(n)}=\partial_{\mu} a_{\nu}^{(n)}-\partial_{\nu} a_{\mu}^{(n)}$ is the 4-dimensional field strength tensor. In above reduction, we need that the $\rho_{n}(z)$ satisfies the following Schrödinger equation

$$
\left[-\partial_{z}^{2}+V_{1}(z)\right] \rho_{n}(z)=m_{n}^{2} \rho_{n}(z)
$$

with the mass-independent potential $V$ is given by

$$
\begin{aligned}
V_{1}(z)=- & \frac{a(a+b) c^{2} \sec ^{2}(\sqrt{a(a+b)} c z)}{16\left[a+b \sin ^{2}(\sqrt{a(a+b)} c z)\right]} \\
\times & \left\{-20 a^{2}-16 a b-10 b^{2}+\left(4 a^{2}+32 a b+11 b^{2}\right) \cos (2 \sqrt{a(a+b)} c z)\right. \\
& \left.-2 b^{2} \cos (4 \sqrt{a(a+b)} c z)+b^{2} \cos (6 \sqrt{a(a+b)} c z)\right\} .
\end{aligned}
$$

The potential also has the asymptotic behavior like that of scalar fields. As same as the discussion about scalar fields, by setting $m_{0}=0$ we can obtain the zero mode

$$
\rho_{0}(z) \propto \mathrm{e}^{\frac{1}{2} A(z)}
$$

which also could not be trapped on the AdS thick brane (see Fig. 母). While, it can be seen from the potential $V_{1}$ that there are infinite bound states for the vector KK modes. By numerical calculation, we can plot the bound states (see Fig. 8) and obtain the mass spectrum:

$$
m_{n}^{2}=\{0.28,1.21,2.34,3.98,5.96,8.37,11.16,14.37,17.97,21.97, \cdots\},
$$

where we have set $a=0.2, b=0.8, r=1, \gamma=-3$. The mass of ground state is $m_{1}=\sqrt{0.28}=0.53$. It is shown that a spin-1 massless vector field is not localized on AdS thick brane. However, massive vector fields can be localized on the AdS thick brane. The mass spectrum (3.36) is plotted in Fig. 9. 


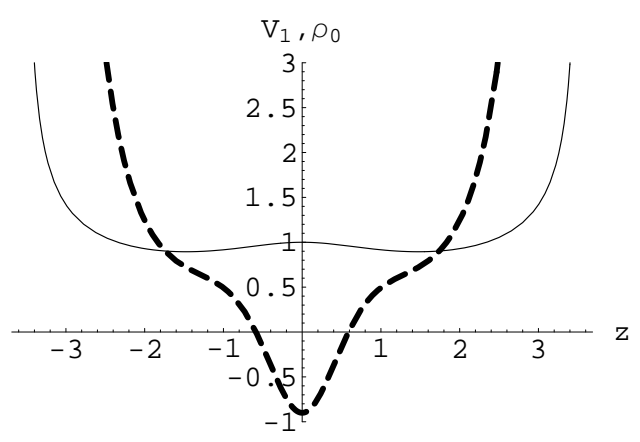

Figure 7: The shapes of the potential $V_{1}(z)$ (the dashed line) and the zero mode $\rho_{0}(z)$ (the thin line). The parameters are set as $a=0.2, b=0.8, r=1, \gamma=-3$.

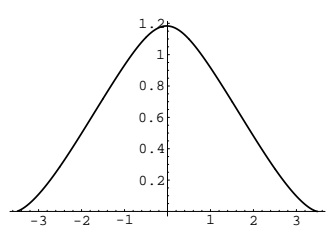

(a) $n=1$

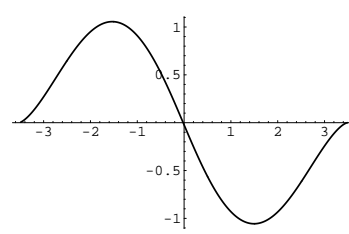

(b) $n=2$

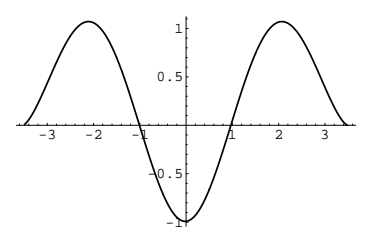

(c) $n=3$

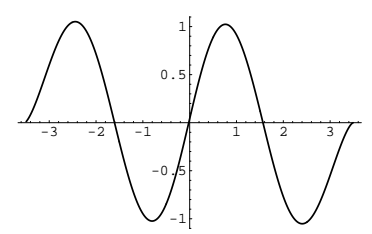

(d) $n=4$

Figure 8: The shapes of the bound KK modes $\rho_{n}(z)$ of vector fields. The parameters are set as $a=0.2, b=0.8, r=1, \gamma=-3$.

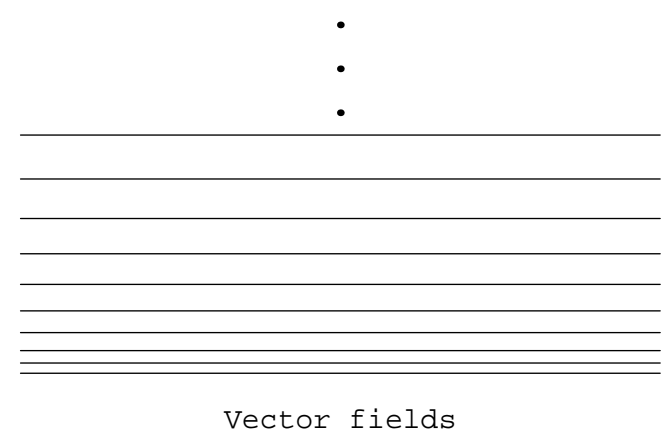

Figure 9: The spectrum $m_{n}^{2}$ of the KK modes of vector fields. The parameters are set as $a=0.2$, $b=0.8, r=1, \gamma=-3$.

\subsection{Spin-1/2 fermion fields}

Finally, we will study localization of fermions on the AdS thick brane. In five dimensions, fermions can be described by four component spinors and their Dirac structure can be described by $\Gamma^{M}=e^{M}{ }_{\bar{M}}^{\bar{M}}$ with $e^{M}{ }_{\bar{M}}$ the vielbein and $\left\{\Gamma^{M}, \Gamma^{N}\right\}=2 g^{M N}$. In this paper, $\bar{M}, \bar{N}, \cdots=0,1,2,3,5$ and $\bar{\mu}, \bar{\nu}, \cdots=0,1,2,3$ denote the $5 \mathrm{D}$ and $4 \mathrm{D}$ local Lorentz indices respectively, and $\Gamma^{\bar{M}}$ are the flat gamma matrices in five dimensions. In our set-up, the 
vielbein is given by

$$
e_{M}^{\bar{M}}=\left(\begin{array}{cc}
\mathrm{e}^{A} \hat{e}_{\mu}^{\bar{\nu}} & 0 \\
0 & \mathrm{e}^{A}
\end{array}\right),
$$

$\Gamma^{M}=\mathrm{e}^{-A}\left(\hat{e}_{\bar{\nu}}^{\mu} \gamma^{\bar{\nu}}, \gamma^{5}\right)=\mathrm{e}^{-A}\left(\gamma^{\mu}, \gamma^{5}\right)$, where $\gamma^{\mu}=\hat{e}_{\bar{\nu}}^{\mu} \gamma^{\bar{\nu}}, \gamma^{\bar{\nu}}$ and $\gamma^{5}$ are the usual flat gamma matrices in the $4 \mathrm{D}$ Dirac representation. The Dirac action of a massless spin- $1 / 2$ fermion coupled to gravity and the background scalar $\phi(y)$ can be expressed as

$$
S_{\frac{1}{2}}=\int d^{5} x \sqrt{-g}\left[\bar{\Psi} \Gamma^{M}\left(\partial_{M}+\omega_{M}\right) \Psi-\eta \bar{\Psi} F(\phi) \Psi\right] .
$$

Here $\omega_{M}$ is the spin connection defined as $\omega_{M}=\frac{1}{4} \omega_{M}^{\bar{M}} \bar{N} \Gamma_{\bar{M}} \Gamma_{\bar{N}}$ and

$$
\begin{aligned}
\omega_{M}^{\bar{M} \bar{N}}= & \frac{1}{2} e^{N \bar{M}}\left(\partial_{M} e_{N}^{\bar{N}}-\partial_{N} e_{M}^{\bar{N}}\right)-\frac{1}{2} e^{N \bar{N}}\left(\partial_{M} e_{N}^{\bar{M}}-\partial_{N} e_{M}^{\bar{M}}\right) \\
& -\frac{1}{2} e^{P \bar{M}} e^{Q \bar{N}}\left(\partial_{P} e_{Q \bar{R}}-\partial_{Q} e_{P \bar{R}}\right) e_{M}^{\bar{R}}
\end{aligned}
$$

The non-vanishing components of the spin connection $\omega_{M}$ for the background metric (3.1) are

$$
\omega_{\mu}=\frac{1}{2}\left(\partial_{z} A\right) \gamma_{\mu} \gamma_{5}+\hat{\omega}_{\mu}
$$

where $\mu=0,1,2,3$ and $\hat{\omega}_{\mu}=\frac{1}{4} \bar{\omega}_{\mu}^{\bar{\mu} \bar{\nu}} \Gamma_{\bar{\mu}} \Gamma_{\bar{\nu}}$ is the spin connection derived from the metric $\hat{g}_{\mu \nu}(x)=\hat{e}_{\mu}^{\bar{\mu}}(x) \hat{e}_{\nu}^{\bar{\nu}}(x) \eta_{\bar{\mu} \bar{\nu}}$. Then we can obtain the equation of motion

$$
\left\{\gamma^{\mu}\left(\partial_{\mu}+\hat{\omega}_{\mu}\right)+\gamma^{5}\left(\partial_{z}+2 \partial_{z} A\right)-\eta \mathrm{e}^{A} F(\phi)\right\} \Psi=0,
$$

where $\gamma^{\mu}\left(\partial_{\mu}+\hat{\omega}_{\mu}\right)$ is the Dirac operator on the brane.

Now we will investigate the 5 -dimensional Dirac equation (3.41), and write the spinor in terms of 4-dimensional effective fields. On account of the Dirac structure of the fifth gamma matrix $\gamma^{5}$, we expect that the left- and right-handed projections of the four dimensional part to behave differently. From the equation (3.41), we will search for the solutions of the general chiral decomposition

$$
\Psi=\mathrm{e}^{-2 A}\left(\sum_{n} \psi_{L n}(x) L_{n}(z)+\sum_{n} \psi_{R n}(x) R_{n}(z)\right),
$$

where $\psi_{L n}(x)=-\gamma^{5} \psi_{L n}(x)$ and $\psi_{R n}(x)=\gamma^{5} \psi_{R n}(x)$ are the left-handed and right-handed components of a 4D Dirac field, respectively. Hence, we assume that $\psi_{L}(x)$ and $\psi_{R}(x)$ satisfy the $4 \mathrm{D}$ massive Dirac equations $\gamma^{\mu}\left(\partial_{\mu}+\hat{\omega}_{\mu}\right) \psi_{L n}(x)=m_{n} \psi_{R_{n}}(x)$ and $\gamma^{\mu}\left(\partial_{\mu}+\right.$ $\left.\hat{\omega}_{\mu}\right) \psi_{R n}(x)=m_{n} \psi_{L_{n}}(x)$. Then the KK modes $L_{n}(z)$ and $R_{n}(z)$ satisfy the following coupled equations

$$
\begin{aligned}
& {\left[\partial_{z}+\eta \mathrm{e}^{A} F(\phi)\right] L_{n}(z)=m_{n} R_{n}(z),} \\
& {\left[\partial_{z}-\eta \mathrm{e}^{A} F(\phi)\right] R_{n}(z)=-m_{n} L_{n}(z) .}
\end{aligned}
$$


From the above coupled equations, we can obtain the Schrödinger-like equations for the left- and right-chiral KK modes of fermions

$$
\begin{aligned}
& \left(-\partial_{z}^{2}+V_{L}(z)\right) L_{n}=m_{L_{n}}^{2} L_{n} \\
& \left(-\partial_{z}^{2}+V_{R}(z)\right) R_{n}=m_{R_{n}}^{2} R_{n}
\end{aligned}
$$

where the mass-independent potentials are given by

$$
\begin{aligned}
V_{L}(z) & =\left(\eta \mathrm{e}^{A} F(\phi)\right)^{2}-\partial_{z}\left(\eta \mathrm{e}^{A} F(\phi)\right), \\
V_{R}(z) & =\left.V_{L}(z)\right|_{\eta \rightarrow-\eta} .
\end{aligned}
$$

For the purpose of getting the standard 4-dimensional action for the massive chiral fermions:

$$
\begin{aligned}
S_{\frac{1}{2}} & =\int d^{5} x \sqrt{-g} \bar{\Psi}\left(\Gamma^{M}\left(\partial_{M}+\omega_{M}\right)-\eta F(\phi)\right) \Psi \\
& =\sum_{n} \int d^{4} x \sqrt{-\hat{g}} \bar{\psi}_{n}\left[\gamma^{\mu}\left(\partial_{\mu}+\hat{\omega}_{\mu}\right)-m_{n}\right] \psi_{n},
\end{aligned}
$$

we need the following orthonormality conditions for $L_{n}$ and $R_{n}$ :

$$
\begin{aligned}
& \int_{-z_{\max }}^{z_{\max }} L_{m} L_{n} d z=\delta_{m n}, \\
& \int_{-z_{\max }}^{z_{\max }} R_{m} R_{n} d z=\delta_{m n}, \\
& \int_{-z_{\max }}^{z_{\max }} L_{m} R_{n} d z=0 .
\end{aligned}
$$

From Eqs. (3.44), (3.45) and (3.46), we can see that, for the left-chiral (right-chiral) fermion localization, there must be some kind of scalar-fermion coupling. This situation is similar to the one in the RS framework [21, 46]. Moreover, if we demand that $V_{L}(z)$ and $V_{R}(z)$ is $Z_{2}$-even with respect to the extra dimension $z, F(\phi)$ should be an odd function of $\phi(z)$. In this paper, we choose the simplest Yukawa coupling: $F(\phi)=\phi$. Then the form of the potentials (3.46) can be expressed as

$$
\begin{aligned}
V_{L}(z)= & \frac{3 \varrho^{4} \eta^{2}\left[a c z+\arctan \left(\frac{\varrho}{a} \tan (\varrho c z)\right)\right]^{2}}{a \cos ^{2}(\varrho c z)\left(a+b \sin ^{2}(\varrho c z)\right)}+\frac{\sqrt{3 a}(a+b) \eta \sec ^{2}(\varrho c z)}{\sqrt{a+(a+b) \tan ^{2}(\varrho c z)}} \\
& \times\left\{-a c-\frac{\varrho c}{a+b \sin ^{2}(\varrho c z)}\left[\varrho+\left(a c z+\arctan \left[\frac{\varrho}{a} \tan (\varrho c z)\right]\right)\right.\right. \\
& \times(a-b \cos (2 \varrho c z) \tan (\varrho c z))]\}
\end{aligned}
$$

where $\varrho \equiv \sqrt{a(a+b)}$. The shapes of the potentials $V_{L, R}(z)$ are shown in Fig. 10 and Fig. 11 for $\eta>\eta_{0}=\frac{2 c}{\sqrt{3} \pi(1+\sqrt{a /(a+b)})}$ and $0<\eta<\eta_{0}$, respectively.

From Fig. 10, it can be seen that, for $\eta>\eta_{0}$, the potentials have the asymptotic behavior: $V_{L, R} \rightarrow+\infty$, when $z \rightarrow \pm z_{\max }$. However, if $0<\eta<\eta_{0}$, the potentials have 

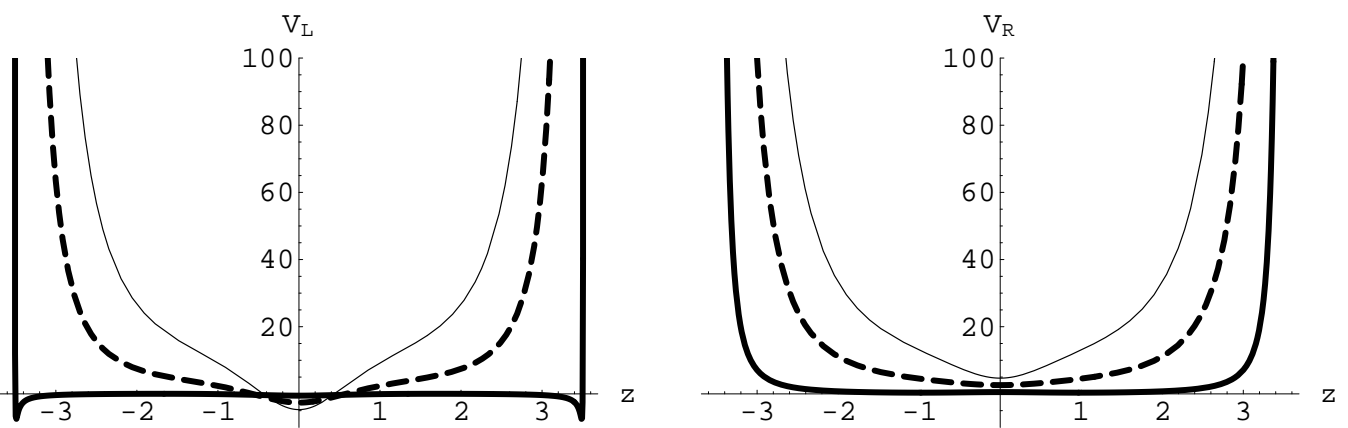

Figure 10: The shapes of the potentials $V_{L}(z)$ and $V_{R}(z)$ for $\eta>\eta_{0}$. The parameters are set as $a=0.2, b=0.8, r=1, \gamma=-3, \eta=0.255$ (the thick line), $\eta=1.254$ (the dashed line), $\eta=2.254$ (the thin line).

different asymptotic behavior: $V_{L} \rightarrow-\infty$ and $V_{R} \rightarrow+\infty$, when $z \rightarrow \pm z_{\max }$ (see Fig. 11). From Fig. 10, it can be seen that, for $\eta>\eta_{0}$, only the potential for left chiral fermions has a negative value at the location of the brane, i.e., $V_{L}(0)<0$ and $V_{R}(0)>0$, so only the zero mode of left chiral fermions could be trapped on the AdS thick brane. Fig. 11 shows that there is no zero mode for both chiral fermions in the case of $0<\eta<\eta_{0}$.
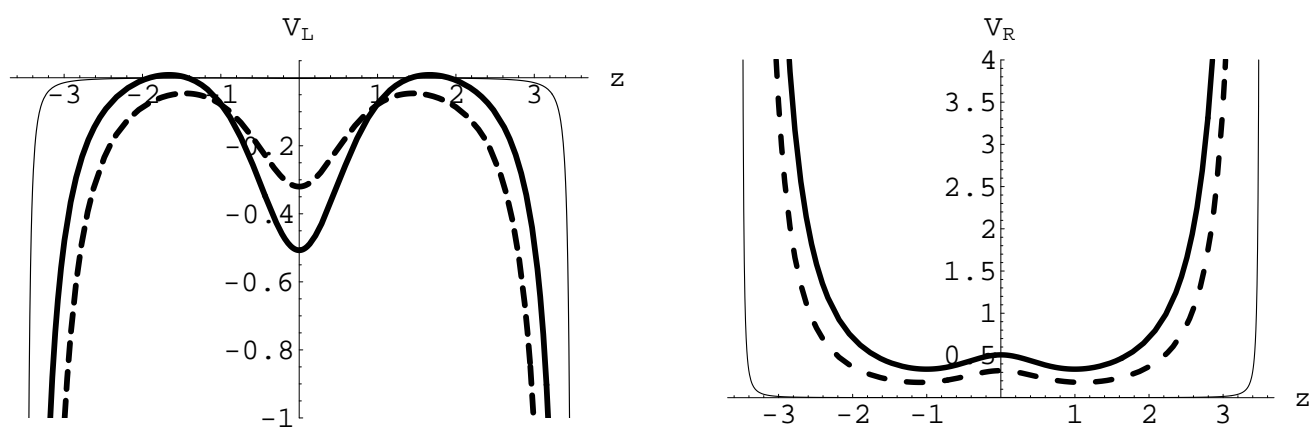

Figure 11: The shapes of the potentials $V_{L}(z)$ and $V_{R}(z)$ for $0<\eta<\eta_{0}$. The parameters are set as $a=0.2, b=0.8, r=1, \gamma=-3, \eta=0.244$ (the thick line), $\eta=0.154$ (the dashed line), $\eta=0.001$ (the thin line).

Setting $\eta>\eta_{0}$, we can obtain the left chiral fermion zero mode solved from (3.43a) by setting $m_{0}=0$ :

$$
L_{0}(z) \propto \exp \left(-\eta \int_{0}^{z} d z^{\prime} \mathrm{e}^{A\left(z^{\prime}\right)} \phi\left(z^{\prime}\right)\right)
$$

This integral is very complex, so we could not give an analytical expression. However, we can plot the shape of zero mode by numerically integral (see Fig. 12).

Furthermore, when $\eta>\eta_{0}$, all the KK states of both left chiral fermions and right chiral fermions are bound states. However, when $\eta<\eta_{0}$, just only the right chiral fermions have infinite bound states. We first consider the case of $\eta>\eta_{0}$. The Schrödinger-like equations for the left and right chiral fermions can be solved numerically, and the bound 


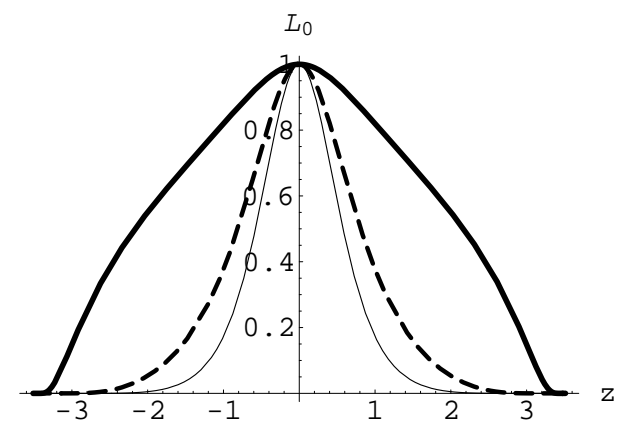

Figure 12: The shapes of the zero mode $L_{0}(z)$ for the case $\eta>\eta_{0}$. The parameters are set as $a=0.2, b=0.8, r=1, \gamma=-3, \eta=0.255$ (the thick line), $\eta=1.254$ (the dashed line), $\eta=2.254$ (the thin line).

states are plotted in Fig. 13 (left chiral fermions) and Fig. 14 (right chiral fermions). The discrete mass spectra for both left and right chiral fermions are calculated as

$$
\begin{aligned}
& m_{L_{n}}^{2}=\{0,4.04,6.77,9.59,12.79,16.39,20.40,24.81,29.64,34.87,40.51, \cdots\},(3.53 \mathrm{a}) \\
& m_{R_{n}}^{2}=\{4.04,6.77,9.59,12.79,16.39,20.40,24.81,29.64,34.87,40.51, \cdots\},(3.53 \mathrm{~b})
\end{aligned}
$$

where the parameters are set as $a=0.2, b=0.8, r=1, \gamma=-3$, and $\eta=1.254$. The ground state of left chiral fermions is the zero mode. However, the ground state of right chiral fermions is massive. The spectra are also shown in Fig. 15.

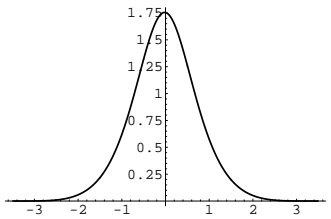

(a) $n=0$

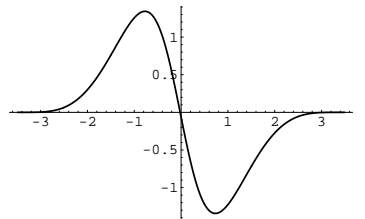

(b) $n=1$

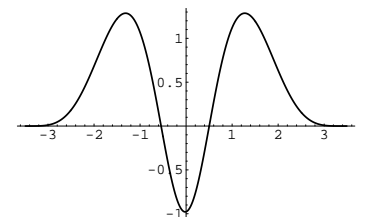

(c) $n=2$

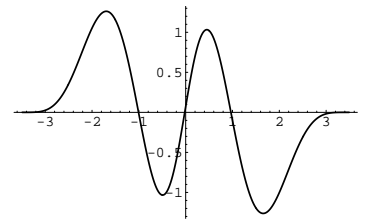

(d) $n=3$

Figure 13: The shapes of the left chiral fermions bound states $L_{n}(z)$ for the case $\eta>\eta_{0}$. The parameters are set as $a=0.2, b=0.8, r=1, \gamma=-3, \eta=1.254$.

Next, we turn to the case $0<\eta<\eta_{0}$. Due to the shape of the potential $V_{L}(z)$, left chiral fermions could not be localized on AdS thick brane. Just only right chiral fermions have bound states. By numerical method, we can obtain these bound states, which are plotted in Fig. 16 for the lower KK states. At the same time, we also get the discrete mass spectrum of right chiral fermions:

$$
m_{R_{n}}^{2}=\{0.51,1.28,2.58,4.26,6.39,8.95,11.95,15.40,19.29,23.62, \cdots\},
$$

with the parameters setting as $a=0.2, b=0.8, c=1$ and $\eta=0.154$. The ground state of right chiral fermions is still massive. The discrete mass spectrum (3.54) is shown in Fig. 17 . 


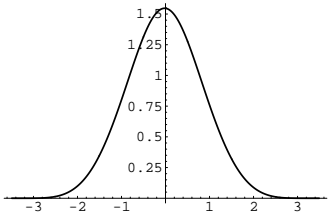

(a) $n=1$

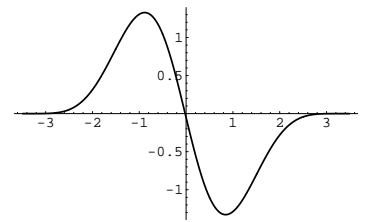

(b) $n=2$

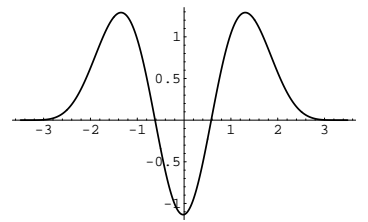

(c) $n=3$

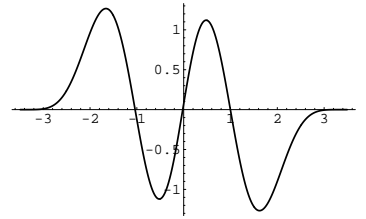

(d) $n=4$

Figure 14: The shapes of the right chiral fermions bound states $R_{n}(z)$ for the case $\eta>\eta_{0}$. The parameters are set as $a=0.2, b=0.8, r=1, \gamma=-3 \eta=1.254$.
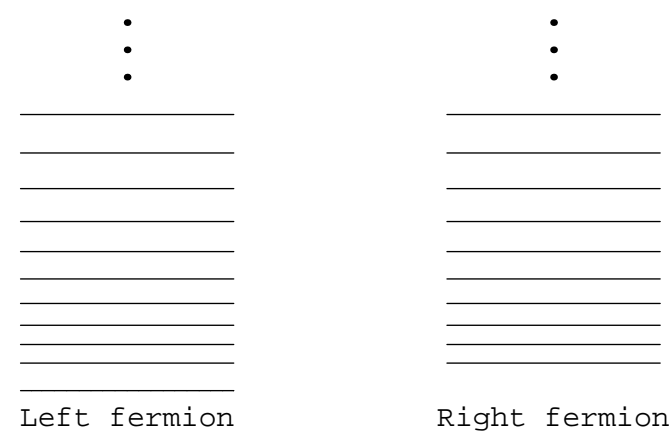

Figure 15: The $m_{n}^{2}$ spectra of left and right chiral fermions for the case $\eta>\eta_{0}$. The parameters are set as $a=0.2, b=0.8, r=1, \gamma=-3, \eta=1.254$.

At the end of this section, we make some comments on the issue of localization of fermions. Localizing fermions on branes requires us to introduce other interactions besides gravity. In our case, when coupling coefficient $\eta=0$, both left and right fermions could not be localized.

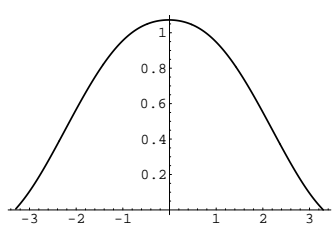

(a) $n=1$

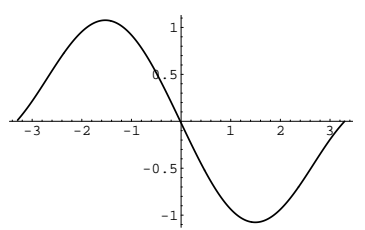

(b) $n=2$

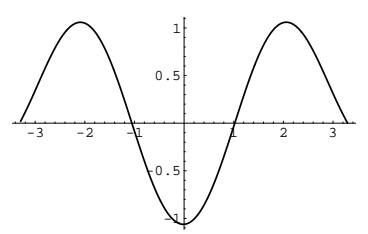

(c) $n=3$

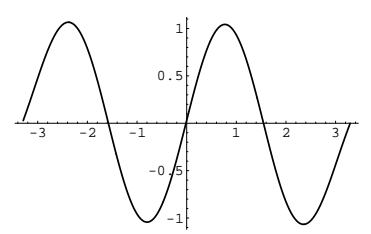

(d) $n=4$

Figure 16: The shapes of the right chiral fermions bound states for the case $0<\eta<\eta_{0}$. The parameters are set as $a=0.2, b=0.8, r=1, \gamma=-3 \eta=0.154$.

\section{Conclusion}

In this paper, we have shown the shapes of the mass-independent potentials of the KK modes of various spin fields in the corresponding Schrödinger equations. In this way, we 


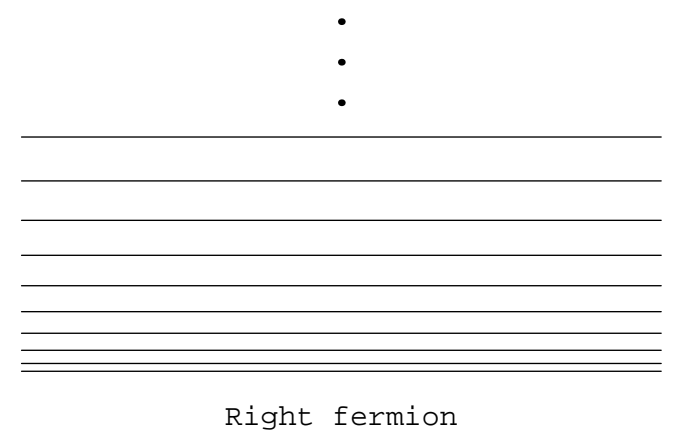

Figure 17: The discrete spectra of right chiral fermions mass for the case $0<\eta<\eta_{0}$. The parameters are set as $a=0.2, b=0.8, r=1, \gamma=-3, \eta=-0.154$.

have investigated the localization and mass spectra of various matters with spin- 0,1 and $1 / 2$ on a kind of AdS thick brane.

For spin-0 scalars, if we do not introduce scalar-scalar couplings $(U=0)$, the situation is same like spin- 1 vectors, both potentials of the KK modes in the corresponding Schrödinger equation have a negative value at the location of the brane, and have the following asymptotic behavior: $V_{0,1}\left(z \rightarrow \pm z_{\max }\right) \rightarrow+\infty$, or equivalently, the potentials trend to infinite when far away from the brane $(y \rightarrow \pm \infty)$. Such potentials suggest that the KK mass spectra of the scalars and vectors are consisted of infinite discrete bound KK modes. However, the massless scalar and vector KK modes could not be trapped on the AdS thick brane. The ground state is massive, and all KK modes are bound states.

When introducing the scalar $\Phi$ coupling with itself and the domain-wall-forming field $\phi$ via a Higgs potential for spin-0 scalars, we found that the coupling constant has a critical value $\lambda_{0}$. We have investigated the cases of $\lambda>\lambda_{0}$ and $\lambda<\lambda_{0}$, respectively. For $\lambda>\lambda_{0}$, the result is same as that of $U=0$, but the massless scalar could be localized by fine-tuning of parameters. However, for $\lambda<\lambda_{0}$, the potential of the KK modes in the corresponding Schrödinger equation has no well, and so there is no bound KK mode. We also have investigated the stability of the solution of $\Phi=0$ inside of the domain wall and far from the domain wall.

For spin-1/2 fermions, if one does not introduce scalar-fermion couplings, there is no bound state for both left- and right-hand KK modes. Hence, we considered the usual Yukawa coupling $\eta \bar{\Psi} \phi \Psi$, and found that the coupling constant $\eta$ has a critical value $\eta_{0}=$ $\frac{2 c}{\sqrt{3} \pi(1+\sqrt{a /(a+b)})}$. For $\eta>\eta_{0}$ and $\eta<\eta_{0}$, the left- and right-hand KK modes have different properties.

For $\eta>\eta_{0}$, just only the potential for the left-chiral fermion KK modes has a finite negative well at the location of the brane, which results in that there exists only the fourdimensional massless left-chiral fermion. However, both potentials of left- and right-chiral fermion KK modes have the same asymptotic behavior: $V_{L, R}\left(z \rightarrow \pm z_{\max }\right) \rightarrow+\infty$. So, both left and right chiral fermion KK modes have infinite bound states. Since the pairs of left-hand and right-hand KK modes couple together through mass terms to become fourdimensional Dirac fermions, the four-dimensional massive Dirac fermions could be localized 
on the AdS brane, and have a set of discrete mass spectrum.

For the situation with $0<\eta<\eta_{0}$, the potential for left-chiral fermion KK modes is very different from the right one. When $z \rightarrow \pm a_{\max }$, the potentials have different asymptotic behavior: $V_{L} \rightarrow-\infty$ and $V_{R} \rightarrow+\infty$. So there is no bound state for left-chiral fermion KK modes, but the spectrum of right-chiral fermion $\mathrm{KK}$ modes is consisted of infinite discrete bound KK modes. Therefor, for the case $0<\eta<\eta_{0}$, no four-dimensional Dirac fermion can be localized on the AdS brane.

\section{Acknowledgement}

This work was supported by the Program for New Century Excellent Talents in University, the National Natural Science Foundation of China (No. 10705013), the Doctoral Program Foundation of Institutions of Higher Education of China (No. 20070730055 and No. 20090211110028), the Key Project of Chinese Ministry of Education (No. 109153), and the Natural Science Foundation of Gansu Province, China (No. 096RJZA055).

\section{References}

[1] V.A. Rubakov and M.E. Shaposhnikov, Do we live inside a domain wall?, Phys. Lett. B 125 (1983) 136; V.A. Rubakov and M.E. Shaposhnikov, Extra space-time dimensions: towards a solution to the cosmological constant problem, Phys. Lett. B 125 (1983) 139; E.J. Squires, Dimensional reduction caused by a cosmological constant, Phys. Lett. B 167 (1986) 286.

[2] M. Visser, An exotic class of Kaluza-Klein models, Phys. Lett. B 159 (1985) 22.

[3] S. Randjbar-Daemi and C. Wetterich, Kaluza-Klein solutions with noncompact internal spaces, Phys. Lett. B 166 (1986) 65.

[4] L. Randall and R. Sundrum, A Large Mass Hierarchy from a Small Extra Dimension, Phys. Rev. Lett. 83 (1999) 3370; An alternative to compactification, Phys. Rev. Lett. 83 (1999) 4690.

[5] J. Lykken and L. Randall, The Shape of Gravity, JHEP 0006 (2000) 014.

[6] I. Antoniadis, A possible new dimension at a few Tev, Phys. Lett. B 246 (1990) 377.

[7] N. Arkani-Hamed, S. Dimopoulos and G. Dvali, The hierarchy problem and new dimensions at a millimeter, Phys. Lett. B 429 (1998) 263; I. Antoniadis, N. Arkani-Hamed, S.

Dimopoulos and G. Dvali, New dimensions at a millimeter to a Fermi and superstrings at a TeV, Phys. Lett. B 436 (1998) 257.

[8] N. Arkani-Hamed, S. Dimopoulos, N. Kaloper and R. Sundtrum, Nucl. Phys. B 480 (2000) 193; S. Kachru, M. Schulz and E. Silverstein, Phys. Rev. D 62 (2000) 045021; A. Kehagias, A conical tear drop as a vacuum-energy drain for the solution of the cosmological constant problem, Phys. Lett. B 600 (2004) 133.

[9] O. DeWolfe, D.Z. Freedman, S.S. Gubser and A. Karch, Modeling the fifth dimension with scalars and gravity, Phys. Rev. D 62 (2000) 046008.

[10] M. Gremm, Four-dimensional gravity on a thick domain wall, Phys. Lett. B 478 (2000) 434; Thick domain walls and singular spaces, Phys. Rev. D 62 (2000) 044017; K. Ghoroku and M. Yahiro, Instability of thick brane worlds, hep-th/0305150; A. Kehagias and K. Tamvakis, A 
Self-Tuning Solution of the Cosmological Constant Problem, Mod. Phys. Lett. A 17 (2002) 1767; Localized Gravitons, Gauge Bosons and Chiral Fermions in Smooth Spaces Generated by a Bounce, Phys. Lett. B 504(2001) 38; M. Giovannini, Gauge-invariant fluctuations of scalar branes, Phys. Rev. D 64 (2001) 064023; Localization of metric fluctuations on scalar branes, Phys. Rev. D 65 (2002) 064008; S. Kobayashi, K. Koyama and J. Soda, Thick brane worlds and their stability, Phys. Rev. D 65 (2002) 064014.

[11] C. Csaki, J. Erlich, T. Hollowood and Y. Shirman, Universal Aspects of gravity localized on thick branes, Nucl. Phys. B 581 (2000) 309.

[12] A. Campos, Critical phenomena of thick brane in warped space-time, Phys. Rev. Lett. 88 (2002) 141602.

[13] A. Wang, Thick de Siter 3branes, dynamic black holes and localization of gravity, Phys. Rev. D 66 (2002) 024024.

[14] R. Emparan, R. Gregory and C. Santos, Black holes on thick branes, Phys. Rev. D 63 (2001) 104022; R. Guerrero, A. Melfo and N. Pantoja, Selfgravitating domain walls and the thin wall limit, Phys. Rev. D 65 (2002) 125010; A. Melfo, N. Pantoja and A. Skirzewski, Thick domain wall space-time with and without reflection symmetry, Phys. Rev. D 67 (2003) 105003; K.A. Bronnikov and B.E. Meierovich, A general thick brane supported by a scalar field, Grav. Cosmol. 9 (2003) 313; O. Castillo-Felisola, A. Melfo, N. Pantoja and A. Ramirez, Localizing gravity on exotic thick three-branes, Phys. Rev. D 70 (2004) 104029.

[15] V. Dzhunushaliev, V. Folomeev, D. Singleton and S. Aguilar-Rudametkin, 6D thick branes from interacting scalar fields, Phys. Rev. D 77 (2008) 044006; V. Dzhunushaliev, V. Folomeev, K. Myrzakulov and R. Myrzakulov, Thick brane in $7 D$ and $8 D$ spacetimes, arXiv:0705.4014; D. Bazeia, F.A. Brito and J.R. Nascimento, Supergravity brane worlds and tachyon potentials, Phys. Rev. D 68 (2003) 085007; D. Bazeia, F.A. Brito and A.R. Gomes, Locally Localized Gravity and Geometric Transitions, JHEP 0411 (2004) 070; D. Bazeia and A.R. Gomes, Bloch Brane, JHEP 0405 (2004) 012; D. Bazeia, F.A. Brito and L. Losano, Scalar fields, bent branes, and RG flow, JHEP 0611 (2006) 064; D. Bazeia, A.R. Gomes and L. Losano, Gravity localization on thick branes: a numerical approach, Int. J. Mod. Phys. A 24 (2009) 1135.

[16] Y.-X. Liu, Y. Zhong and K. Yang, Scalar-Kinetic Branes, arXiv:0907.1952[hep-th].

[17] R. Davies, D. P. George, and R. R. Volkas, Standard model on a domain-wall brane? , Phys. Rev. D 77 (2008) 124038.

[18] D. P. George, M. Trodden and R. R. Volkas, Extra-dimensional cosmology with domain-wall branes, JHEP 0902 (2009) 035.

[19] R. Davies and D. P. George, Fermions, scalars, and Randall-Sundrum gravity on domain-wall branes, Phys. Rev. D 76 (2007) 104010; D. P. George and R. R. Volkas, Kink modes and effective four dimensional fermion and Higgs brane models, Phys. Rev. D 75 (2007) 105007.

[20] V. Dzhunushaliev, V. Folomeev and M. Minamitsuji, Thick brane solutions, arXiv:0904.1775[gr-qc].

[21] B. Bajc and G. Gabadadze, Localization of matter and cosmological constant on a brane in anti de Sitter space, Phys. Lett. B 474 (2000) 282.

[22] I. Oda, Localization of matters on a string-like defect, Phys. Lett. B 496 (2000) 113. 
[23] Y.-X. Liu, X.-H. Zhang, L.-D. Zhang and Y.-S. Duan, Localization of Matters on Pure Geometrical Thick Branes, JHEP 0802 (2008) 067; Y.-X. Liu, L.-D. Zhang, L.-J. Zhang and Y.-S. Duan, Fermions on Thick Branes in the Background of Sine-Gordon Kinks, Phys. Rev. D 78, 065025 (2008).

[24] Y. Grossman and N. Neubert, Neutrino masses and mixings in non-factorizable geometry, Phys. Lett. B 474 (2000) 361; R. Koley and S. Kar, A novel braneworld model with a bulk scalar field, Phys. Lett. B 623 (2005) 244. [Erratum ibid. 631 (2005) 199; A. Melfo, N. Pantoja and J.D. Tempo, Fermion localization on thick branes, Phys. Rev. D 73 (2006) 044033.

[25] T.R. Slatyer and R.R. Volkas, Cosmology and fermion confinement in a scalar-field generated domain wall brane in five dimensions, JHEP 0704 (2007) 062;

[26] S. Ichinose, Fermions in Kaluza-Klein and Randall-Sundrum theories, Phys. Rev. D 66 (2002) 104015.

[27] C. Ringeval, P. Peter and J.P. Uzan, Localization of massive fermions on the brane, Phys. Rev. D 65 (2002) 044016.

[28] T. Gherghetta and M. Shaposhnikov, Localizing gravity on a string-like defect in six dimensions, Phys. Rev. Lett. 85 (2000) 240.

[29] I.P. Neupane, Consistency of higher derivative gravity in the brane background, JHEP 0009 (2000) 040; I.P. Neupane, Completely localized gravity with higher curvature terms, Class. Quant. Grav. 19 (2002) 5507.

[30] S. Randjbar-Daemi and M. Shaposhnikov, Fermion zero-modes on brane-worlds, Phys. Lett. B $492(2000) 361$.

[31] R. Koley and S. Kar, Scalar kinks and fermion localisation in warped spacetimes, Class. Quant. Grav. 22 (2005) 753.

[32] S.L. Dubovsky, V.A. Rubakov and P.G. Tinyakov, Brane world: disappearing massive matter, Phys. Rev. D 62 (2000) 105011.

[33] Y. Brihaye and T. Delsate, Remarks on bell-shaped lumps: stability and fermionic modes, Phys. Rev. D 78 (2008) 025014.

[34] Y.X. Liu, L. Zhao and Y.S. Duan, Localization of Fermions on a String-like Defect, JHEP 0704 (2007) 097; Y.X. Liu, L. Zhao, X.H. Zhang and Y.S. Duan, Fermions in Self-dual Vortex Background on a String-like Defect, Nucl. Phys. B 785 (2007) 234; Y.Q. Wang, T.Y. $\mathrm{Si}$, Y.X. Liu and Y.S. Duan, Fermionic zero modes in self-dual vortex background, Mod. Phys. Lett. A 20 (2005) 3045; L. Zhao, Y.-X. Liu and Y.i-S. Duan, Fermions in gravity and gauge backgrounds on a brane world, Mod. Phys. Lett. A 23 (2008) 1129.

[35] C.A.S. Almeida, R. Casana, M.M. Ferreira and A.R. Gomes, Fermion localization and resonances on two-field thick branes, Phys. Rev. D 79 (2009) 125022.

[36] Y.-X. Liu, J. Yang, Z.-H. Zhao, C.-E. Fu and Y.-S. Duan, Fermion Localization and Resonances on A de Sitter Thick Brane, to appear in Phys. Rev. D, arXiv:0904.1785[hep-th]; Y.-X. Liu, C.-E. Fu, L. Zhao and Y.-S. Duan, Localization and Mass Spectra of Fermions on Symmetric and Asymmetric Thick Branes, to appear in Phys. Rev. D, arXiv:0907.0910[hep-th].

[37] R. Koley, J. Mitra and S. SenGupta, Fermion localization in a generalized Randall-Sundrum model, Phys. Rev. D 79 (2009) 041902(R). 
[38] Y. Kodama, K. Kokubu and N. Sawado, Localization of massive fermions on the baby-skyrmion branes in 6 dimensions, Phys. Rev. D 79, 065024 (2009).

[39] Y.-X. Liu, H.-T. Li, Z.-H. Zhao, J.-X. Li and J.-R. Ren, Fermion Resonances on Multi-field Thick Branes, JHEP 0910 (2009) 091; Z.-H. Zhao, Y.-X. Liu and H.-T. Li, Fermions on Asymmetric Bloch Branes, arXiv:0911.2572; Y.-X. Liu, K. Yang and Y. Zhong, de Sitter Thick Brane Solution in Weyl Geometry, arXiv:0911.0269.

[40] O. Arias, R. Cardenas and I. Quiros, Thick Brane Worlds Arising From Pure Geometry, Nucl. Phys. B 643 (2002) 187; N. Barbosa-Cendejas and A. Herrera-Aguilar, 4D gravity localized in non $Z_{2}$-symmetric thick branes JHEP 0510 (2005) 101; N. Barbosa-Cendejas and A. Herrera-Aguilar, Localization of 4 D gravity on pure geometrical thick branes, Phys. Rev. D 73 (2006) 084022.

[41] N. Barbosa-Cendejas, A. Herrera-Aguilar, M. A. ReyesSantos and C. Schubert, Mass gap for gravity localized on Weyl thick branes, Phys. Rev. D 77 (2008) 126013; N. Barbosa-Cendejas, A. Herrera-Aguilar, U. Nucamendi and I. Quiros, Mass hierarchy and mass gap on thick branes with Poincare symmetry, arXiv:0712.3098[hep-th].

[42] Y.-X. Liu, Z.-H. Zhao, S.-W. Wei and Y.-S. Duan, Bulk Matters on Symmetric and Asymmetric de Sitter Thick Branes, JCAP 02 (2009) 003.

[43] Y.-X. Liu, L.-D. Zhang, S.-W. Wei and Y.-S. Duan, Localization and Mass Spectrum of Matters on Weyl Thick Branes, JHEP 0808 (2008) 041.

[44] S. Kobayashi, K. Koyama and J. Soda, Thick brane worlds and their stability, Phys. Rev. D 65 (2002) 064014.

[45] A. Karch and L. Randall, Locally Localized Gravity, JHEP 0105 (2001) 008.

[46] R. Jackiw and C. Rebbi, Solitons with fermion number 1/2, Phys. Rev. D 13 (1976) 3398. 